\title{
Oil flow damage in Kraft paper 2: UV irradiation
}

\author{
Ian L. Hosier, \\ Tony Davies High Voltage \\ Laboratory, \\ University of Southampton, \\ Southampton, SO17 1BJ, UK \\ ilh@soton.ac.uk
}

\author{
Paul L. Lewin, \\ Tony Davies High Voltage \\ Laboratory, \\ University of Southampton, \\ Southampton, SO17 1BJ, UK \\ pll@ecs.soton.ac.uk
}

\author{
Gordon Wilson, \\ National Grid, \\ Warwick Technology Park, \\ Gallows Hill, \\ Warwick, CV34 6DA, UK \\ gordon.wilson@nationalgrid.com
}

\begin{abstract}
Ultraviolet (UV) irradiation was used as a tool to degrade Kraft paper. Using measurements of the DP as a function of irradiation time, a theoretical model was used to predict the local value of DP in the surface layer of the paper. After characterization of their mechanical properties, selected samples were exposed to oil flows mimicking ONAN, OFAF and reclamation conditions. The presence of a surface layer of low DP led to significant surface roughening under high rates of oil flow, but no significant erosion. The findings indicate that the DP value at the surface is more critical than the average (or measured) DP in determining the extent of oil flow related damage.
\end{abstract}

Keywords-Kraft paper, UV aging, DP, mineral oil

\section{INTRODUCTION}

Industry based studies have shown that a major cause of faults in high voltage transformers is mechanical failure of the paper, therefore monitoring its condition is crucial for determining plant health [1]. A direct indicator of the condition of Kraft paper is the degree of polymerisation (DP) and typically new Kraft has a DP of $\sim 1100$, a value of $\sim 200$ is taken to indicate end of life [2], whilst values of 300 - 500 are more typical of an aged asset [3]. As the DP value falls, the paper becomes mechanically brittle [1] and so it is easy to envisage scenarios where aged paper can be eroded by oil flow. For instance, switching from natural cooling (ONAN) to forced cooling (OFAF/ODAF) can increase the oil flow rate considerably [4, 5]. Of more concern are the high flow rates $(>2000 \mathrm{l} / \mathrm{h})$ inherent to routine reclamation activities [6] so, having an understanding of the consequences of imparting oil flow over a paper surface with a known DP value, will assist network operators to make better asset management decisions.

Exposure to ultraviolet (UV) light can have many effects on paper, it can sever molecular bonds leading to a reduction in DP [7] and conservationists are familiar with its effects in yellowing and embrittling precious manuscripts [8]. Since oil flow affects only the surface layers of the paper [9], it is only necessary to degrade this region and UV irradiation provides an energy efficient way of achieving this. In this regard short wavelengths $(<430 \mathrm{~nm})$ are more applicable due to their higher potential for molecular damage and smaller penetration depth [10].

In this investigation, which forms part of an ongoing project exploring the relationships between aging and mechanical damage when Kraft paper is exposed to oil flows mimicking those found in plant, Kraft paper was degraded through exposure to UV irradiation. Comparison of measured DP values to a theoretical model enabled the local DP at the crucial surface layer to be calculated. Selected samples were then taken and exposed to oil flows simulating ONAN, OFAF and reclamation conditions. The resulting surface damage was assessed through optical microscopy, whilst measurements of sample thickness allowed any flow related erosion to be quantified.

\section{METHODOLOGY}

\section{A. Paper aging and characterisation}

Transformer grade Kraft paper (0.1 mm thickness) was cut into $10 \times 10 \mathrm{~cm}$ pieces and placed into a bespoke UV unit for irradiation. This kit was comprised of a $300 \mathrm{~mm}$ long T5/8 W tube driven by an electronic ballast (Osram), this was centered within a $68 \mathrm{~mm}$ diameter PVC pipe and the paper samples (four in total) were placed carefully onto its inside surface. This arrangement ensures a constant distance between the entire surface of the paper and the light source. For UVC irradiation (254 nm) a germicidal tube was used, whilst for UVA irradiation $(368 \mathrm{~nm})$ an insect killer tube was used. At the surface of the paper, the tubes give radiant intensities of 4.1 and $3.8 \mathrm{~mW} / \mathrm{cm}^{2}$ respectively.

DP measurements were then performed according to ASTM D4243 using Bis(ethylenediamine) copper (II) hydroxide (Sigma Aldrich) as solvent [2] and performing the required viscosity measurements in a glass viscometer tube $(\mathrm{C}=0.01)$ at $20.0 \pm 0.5^{\circ} \mathrm{C}$. Tensile testing was then undertaken using a Tinius Olsen $\mathrm{H} 25 \mathrm{KS}$ tensiometer on cut strips (nominally $70 \mathrm{x}$ $15 \mathrm{~mm}$ ) using a gauge length of $50 \mathrm{~mm}$ and a crosshead speed of $10 \mathrm{~mm} / \mathrm{min}$.

\section{B. Oil flow testing}

Before flow testing, all samples were vacuum impregnated with new mineral oil (Nynas Gemini X) in glass jars. A bespoke oil flow tester was constructed and full details of the design are provided in our previous paper [9], but for completion, images of the equipment are provided in Fig. 1. The equipment (Fig. 1a) comprises a heated mineral oil-filled tank and a circulation pump. The samples to be tested are attached to a tilted plate (Fig. 1b) and in this image, oil enters from the left, passes over the sample and is drawn out from the back of the tank. The heaters are mounted behind the sample holder to avoid distorting the oil flow over the sample. An identical testing protocol was 

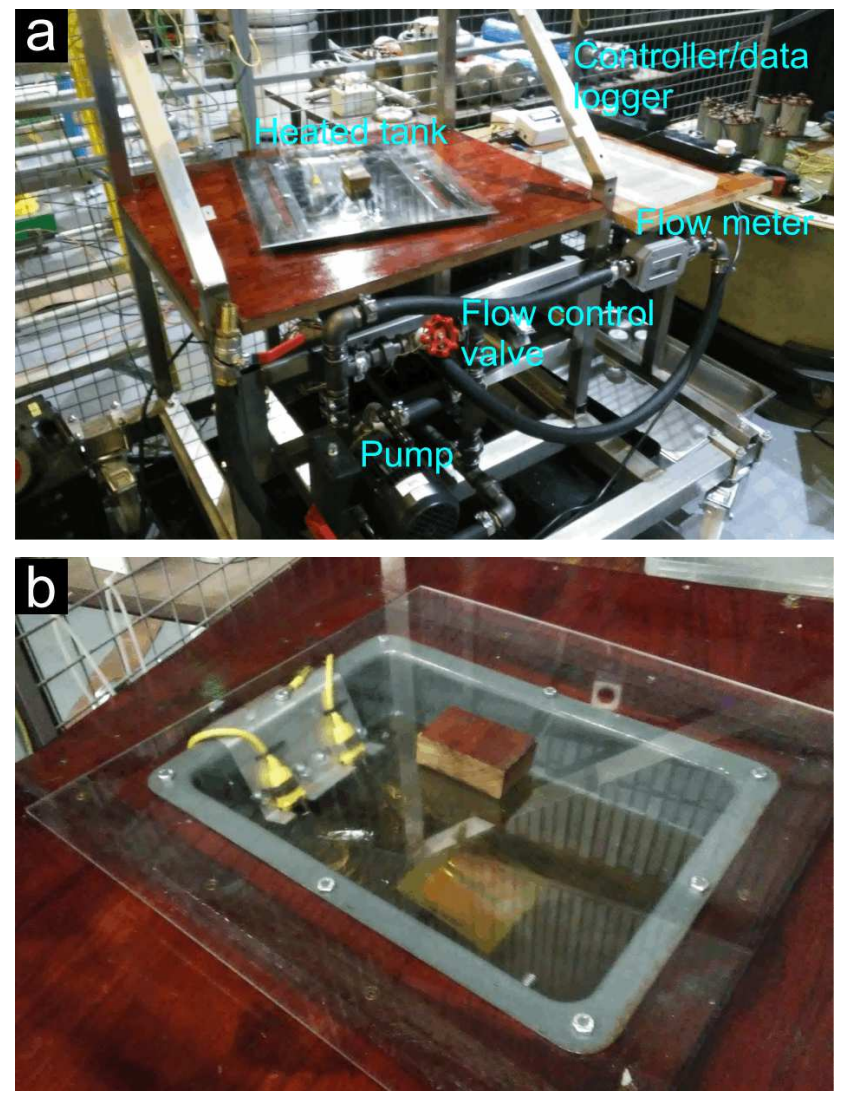

Fig. 1. Oil flow equipment; (a) overview indicating main components, (b) details of oil tank showing tilted plate sample holder.

used as for the thermal aged samples [9]; tests were performed at $40{ }^{\circ} \mathrm{C}$ using new mineral oil and flow rates of 3,12 and 33 $1 /$ min to simulate ONAN, OFAF and reclamation conditions respectively. A duration of $6 \mathrm{~h}$ was used to allow sufficient time for the oil to warm up and to fit the test into the working day.

\section{Characterisation of flow related damage}

Following flow testing, samples were submerged in a bath of acetone for at least $1 \mathrm{~h}$ and then left on absorbent material to dry. Optical microscopy was then performed under oblique illumination and finally, ten independent measurements of thickness were taken and the data analysed in Microsoft Excel.

\section{UV aging model}

Experimental measurements of DP yield an average value for the entire thickness of the paper however, UV exposure affects the surface layers more than the bulk, leaving a surface layer with a lower DP [7]. It is this value of DP, rather than the average value, which will govern the extent of oil flow related surface damage. Since it is impractical to scrape off the surface layer and measure its DP directly, a simple theoretical model was devised to calculate the depth and time dependence of DP from first principles. Cumulative damage to the paper, expressed as the number $n$ of chain scissions, can be written in terms of the DP of each fragment and that of the undamaged sample $\left(D P_{o}\right)$ as;

$$
n=\frac{D P_{0}}{D P}-1
$$

We will assume a simple exponential decay of intensity $I$ with depth $d$ into the paper (Beer-Lambert law). For a source of intensity $I_{o}$;

$$
I=I_{o} e^{-d / \lambda}
$$

Where $\lambda$ is the "penetration depth" and depends on wavelength. Next, assuming a fraction $\alpha$ of the radiation causes chain scissions and that the damage is cumulative with time $t$, at a depth $d$ into the paper we can write;

$$
n(d)=\alpha I_{o} t e^{-d / \lambda}
$$

By equating eq. 1 and eq. 3 we get the expression;

$$
\frac{D P_{o}}{D P}=1+\alpha I_{o} t e^{-d / \lambda}
$$

Rearranging for DP we find;

$$
D P=\frac{D P_{o}}{1+\alpha I_{o} t e^{-d / \lambda}}
$$

In actuality, the DP value never goes to zero as $t \rightarrow \infty$ as suggested by the equation above, but approaches a finite value $\left(D P_{\min }\right)$ [2]. To reflect this, the boundary conditions were changed to yield the final expression;

$$
D P=\frac{\left(D P_{o}-D P_{\min }\right)}{1+\alpha I_{o} t e^{-d / \lambda}}+D P_{\text {min }}
$$

Values of $\lambda$ for UVC and UVA irradiation can be obtained from the literature [10] whilst $\alpha$ can be determined by fitting to experimental data. Obtaining a value that can be compared to the experimentally measured DP from the above equation, involves averaging the calculated DP values over the entire thickness of the paper; this was done numerically.

\section{RESULTS}

\section{A. Initial characterisation of aged samples}

Fig. 2a shows average values of DP obtained from three independent measurements. DP falls with irradiation time and UVC is more effective than UVA as anticipated [7, 10]. The model (eq. 6) was fitted to the data starting with reasonable values of $D P_{o}, D P_{\min }$ and $\lambda$, whilst varying $\alpha$. Despite the simplicity of the model, the fit is remarkably good and the value of $\alpha$ for UVC is around 9 times the value for UVA, which is in good agreement with the literature (see Fig. 6.2 in [10]).

With suitable parameters now established, the model can be used to predict the depth and time dependence of DP. Predictions for UVC exposure are shown in Fig. $2 b$ and as expected, the DP is lower at the exposed surface $(d=0)$ of the paper than in the bulk. Very short irradiation times are needed to produce samples with surface DP values consistent with either, aged assets $(\leq 48 \mathrm{~h})$ or end of life conditions $(\geq 96 \mathrm{~h})$. Therefore, compared to thermal aging, UV irradiation represents an energy efficient and time saving way of producing degraded paper samples for oil flow testing.

Average values of break strain obtained from three independent tensile tests are shown in Fig. 2c. For new paper the break strain in the longitudinal or fibre direction (L) is lower than in the transverse $(\mathrm{T})$ direction, reflecting the expected anisotropy [1]. UVC exposure has the same effect as thermal aging [9]; the break strain is progressively reduced in both 

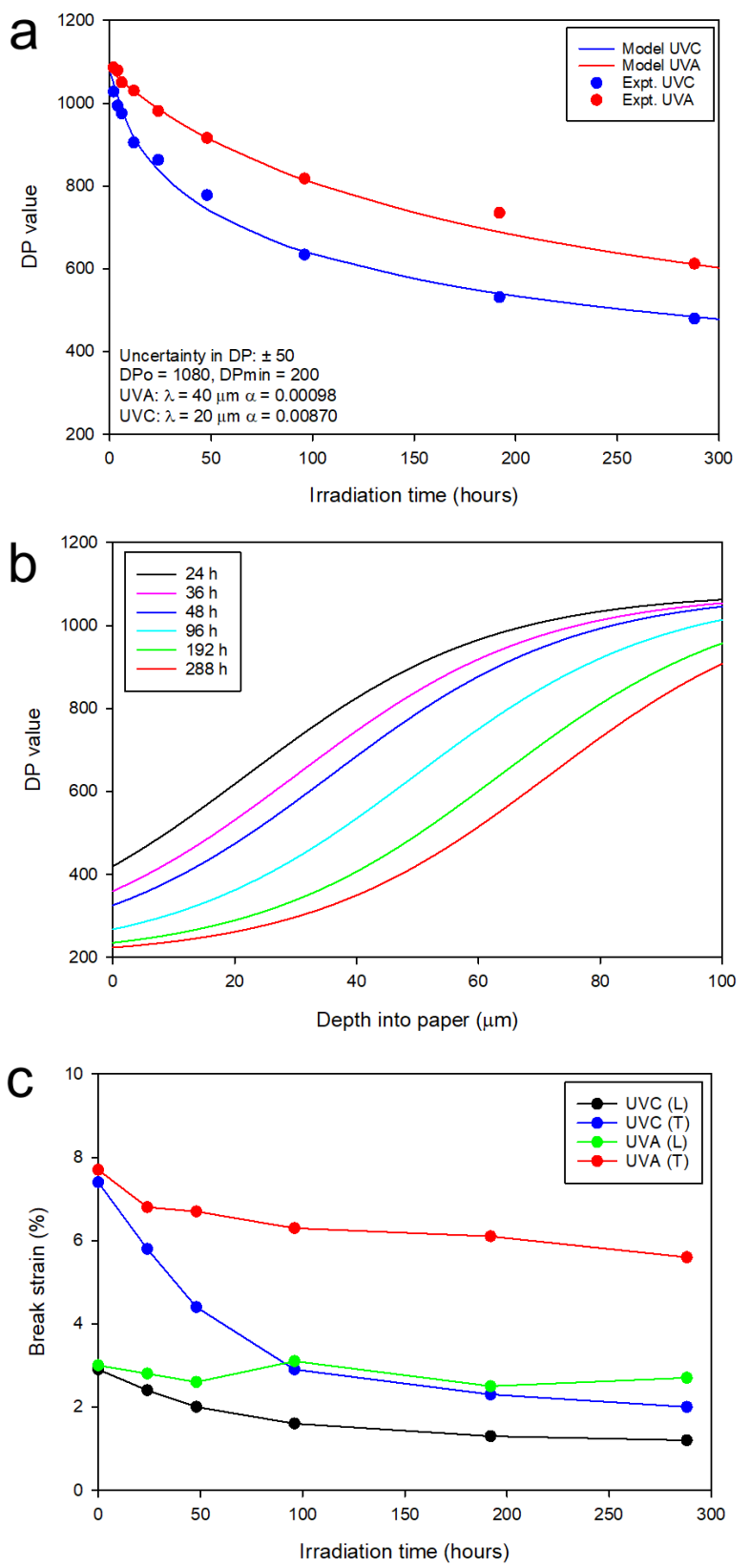

Fig. 2. Initial characterisation of UV irradiated samples (a) measured DP values with fit to theoretical model, (b) predicted DP as a function of depth into the paper for UVC irradiation, (c) measurements of break strain

measuring directions and the paper becomes brittle. In contrast, UVA irradiation has much less of an effect and only seems to reduce the transverse break strain. This indicates that long wavelength radiation only affects the lignin and leaves the fibres relatively untouched [8]. Samples irradiated under UVC for 36, 96 and $288 \mathrm{~h}$ were therefore selected for flow testing. These represent a good spread of average DP values between 850 and 500 (Fig. 2a) and the surface DP values of 370, 270 and 220 (Fig. 2b) conveniently represent aged assets to end of life [2,3].

\section{B. Observed surface damage due to oil flow}

As in the case of thermal aging [9], three distinct morphologies could be identified following oil flow testing and there was no discernible effect of flow direction on the surface
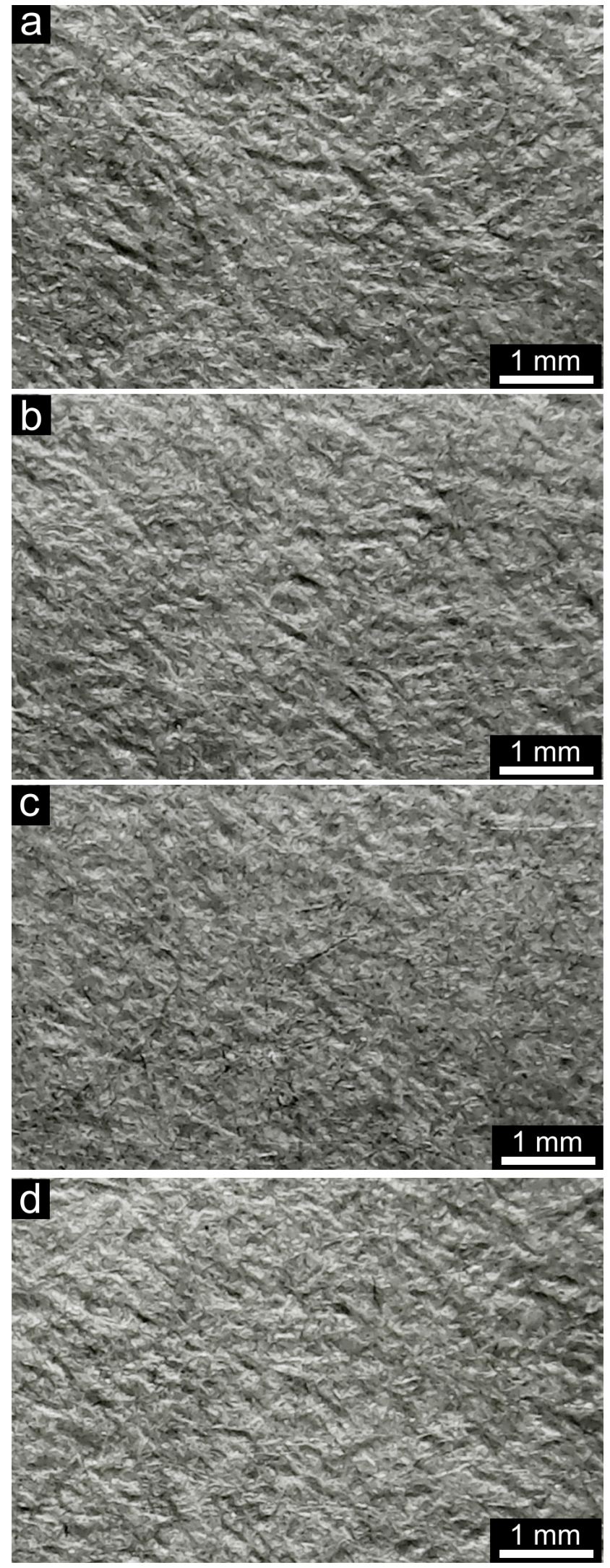

Fig. 3. Optical microscopy (a) $96 \mathrm{~h}$ irradiation, ONAN conditions, (b) 96 $\mathrm{h}$ irradiation, OFAF conditions, (c) $36 \mathrm{~h}$ irradiation, reclamation conditions, (d) $288 \mathrm{~h}$ irradiation, reclamation conditions 
textures. All three samples exposed to ONAN conditions showed the texture of Fig. 3a; deep furrows (arising from the rolling process) are evident which are oriented along the fibre direction (top left to bottom right); this indicates that no significant surface damage has occurred - blue ranking (Table 1). Meanwhile, all samples exposed to OFAF conditions show a "worn" appearance (Fig. 3b) and noticeable surface damage has occurred as indicated by less distinct furrowing - green ranking. Finally, all three samples under reclamation conditions, have the "random" textures shown in Figs. $3 \mathrm{c}$ and $3 \mathrm{~d}$ - red ranking. Here the lignin appears to have been completely removed by the oil flow leaving the underlying fibres exposed.

Comparison of Table 1 below to the equivalent colour coded ranking table for thermally aged samples [9], shows that the current samples follow the ranking scheme of thermally aged samples that have been aged for $14 \mathrm{~d}$ or more (DP $<350)$. That is, reclamation conditions always produce significant surface damage (red ranking) even after $36 \mathrm{~h}$ irradiation (Fig. 3c). This confirms that the surface, rather than the average, DP controls the extent of surface damage in these UV irradiated samples.

TABLE I. RANKING OF SAMPLES FROM OIL FLOW TESTING AT $40{ }^{\circ} \mathrm{C}$

\begin{tabular}{|c|c|c|c|c|}
\hline \multirow{2}{*}{$\begin{array}{l}\text { Irradiation } \\
\text { time }\end{array}$} & \multirow{2}{*}{ Orientation } & \multicolumn{3}{|c|}{ Oil flow rate } \\
\hline & & $O N A N$ & $O F A F$ & Reclamation \\
\hline \multirow{2}{*}{$36 \mathrm{~h}$} & Longitudinal & & & \\
\hline & Transverse & & & Fig. 3c \\
\hline \multirow{2}{*}{$96 \mathrm{~h}$} & Longitudinal & Fig. 3a & & \\
\hline & Transverse & & Fig. 3b & \\
\hline \multirow{2}{*}{$288 \mathrm{~h}$} & Longitudinal & & & \\
\hline & Transverse & & & Fig. 3d \\
\hline
\end{tabular}

\section{Measurements of sample thickness}

Fig. 4 shows a histogram of average thickness values for new paper and the three irradiated papers $(36,96$ and $288 \mathrm{~h})$, three flow rates (ONAN, OFAF and reclamation) and two orientations (L or T). Unlike thermal aging [9], UV exposure does not appear to change the average thickness of the samples which remained at $\sim 95 \mu \mathrm{m}$ throughout. A statistical comparison (t-test) of all the data within each group (i.e. any fixed aging time), did not

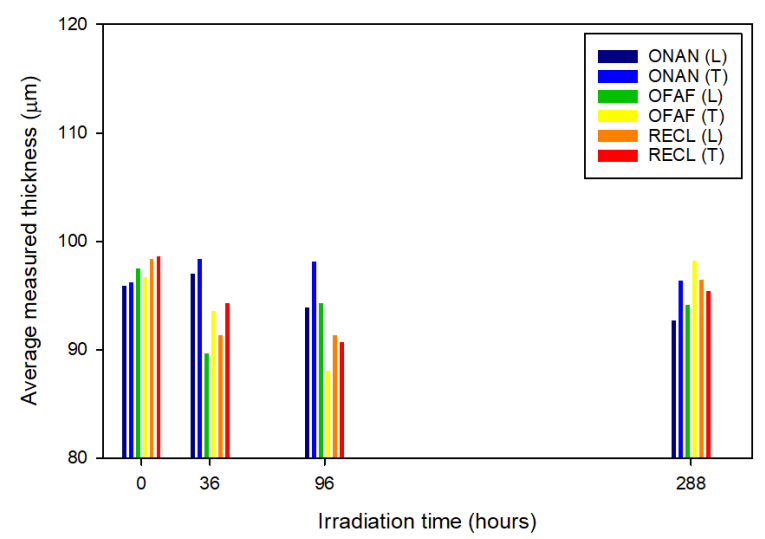

Fig. 4. Average thickness values as a function of flow conditions, orientation and irradiation time reveal any significant erosion. Therefore, the morphological changes are confined to the surface layers of the paper $(<2 \mu \mathrm{m})$.

\section{CONCLUSIONS}

Kraft paper was irradiated under UV light and it's DP and mechanical properties were characterised. A theoretical model allowed the DP at the critical surface layer of the paper to be calculated. UVC irradiation was found to be an energy efficient and time saving way of preparing paper samples with a low value of surface DP, which emulate the condition of the paper which would be found in a severely aged asset.

Selected samples were exposed to oil flow rates mimicking ONAN, OFAF and reclamation conditions. The surface damage was identical to thermally aged paper exposed to a high degree of aging, despite their relatively high measured DP ( $>500)$. This confirmed that the much lower DP at the surface controls the extent of oil flow related damage. This preliminary work shows that the insulation system of heavily aged assets is unlikely to be damaged by normal reclamation activities. Longer duration tests at higher oil temperatures are currently underway to confirm this finding, and will be reported in due course.

\section{ACKNOWLEDGMENT}

The authors would like to acknowledge National Grid Electricity Transmission for supporting the project and agreeing to the publication of the results. The "TOPICS2" project (part of NIA_NGET0214) was made possible through the Network Innovation Allowance.

\section{REFERENCES}

[1] A. M. Emsley, R. J. Heywood, M. Ali, and X. Xiao, "Degradation of cellulosic insulation in power transformers. Part 4: Effects of ageing on the tensile strength of paper," IEEE Proc. Sci. Meas. Tech., vol. 147, pp. 285-290, 2000.

[2] R. J. Heywood, A. M. Emsley, M. Ali, "Degradation of cellulose insulation in power transformers. Part 1: Factors affecting the measurement of the average viscometric degree of polymerisation of new and aged electrical papers", IEEE Proc. Meas. Technol., vol. 147, pp. 86 - 90, 2000.

[3] N. Lelekakis, W. Guo, D. Martin, J. Wijaya, D. Susa, "A Field Study of Aging in Paper-Oil Insulation Systems", IEEE Electr. Insul. Mag., vol. 28, pp. $12-19,2012$.

[4] J. Gastelurrutia, J. C. Ramos, G. S. Larraona, A. Rivas, J. Izagirre, L. del Rio, "Numerical modelling of natural convection of oil inside distribution transformers", Appl. Therm. Eng., vol. 31, pp. 493-505, 2011.

[5] H. Campelo, C. M. Fonte, R.G. Sousa, J. C. B. Lopes, R. Lopes, J. Ramos, D. Couto, M. M. Dias, "Detailed CFD analysis of ODAF power transformers", In Proc. Intern. Coll. Transf. Res. Asset. Manag. (2009), DOI: $10.13140 / 2.1 .2854 .2402$.

[6] J. S. N'Cho, I. Fofana, A. Beroual, T. Aka-Ngnui, J. Sabau, "Aged Oils Reclamation: Facts and Arguments Based on Laboratory Studies", IEEE Trans. Diel. Electr. Insul., vol. 19, pp. 1583-1592, 2012.

[7] J. H. Flynn, W. K. Wilson, W. L. Morrow, "Degradation of cellulose in a vacuum with ultraviolet light”, J. Res. Nat. Bur. Stand., vol. 60, pp. 229 233, 1958.

[8] R. L. Feller in "Accelerated Aging: photochemical and physical aspects", Ed. D. Berland, J. Paul Getty Trust, Michigan, USA, 1994.

[9] I. L. Hosier, P. L. Lewin, G. Wilson, "Oil flow damage in Kraft paper 1: thermal aging", 2021 Electr. Insul. Conf., submitted.

[10] Y. Kataoka, M. Kiguchi, R. S. Williams, P. D. Evans, "Violet light causes photodegradation of wood beyond the zone affected by ultraviolet radiation", Holzforschung, vol. 61, pp. 23-27, 2007. 